\title{
Exploring of Determinants Factors of Anti-Diabetic Medication Adherence in Several Regions of Asia - A Systematic Review
}

\author{
Much Ilham Novalisa Aji Wibowo $\mathbb{D}^{1,2}$, Nanang Munif Yasin ${ }^{3}$, Susi Ari Kristina ${ }^{4}$, Yayi Suryo Prabandari ${ }^{5}$ \\ 'Doctoral Program in Pharmaceutical Science, Faculty of Pharmacy, Universitas Gadjah Mada, Yogyakarta, Indonesia; ${ }^{2}$ Department of Pharmacy, \\ Faculty of Pharmacy, Universitas Muhammadiyah Purwokerto, Purwokerto, Indonesia; ${ }^{3}$ Department of Pharmacology and Clinical Pharmacy, Faculty of \\ Pharmacy, Universitas Gadjah Mada, Yogyakarta, Indonesia; ${ }^{4}$ Department of Pharmaceutics, Faculty of Pharmacy, Universitas Gadjah Mada, Yogyakarta, \\ Indonesia; ${ }^{5}$ Department of Public Health, Faculty of Medicine, Public Health, and Nursing, Universitas Gadjah Mada, Yogyakarta, Indonesia \\ Correspondence: Nanang Munif Yasin, Department of Pharmacology and Clinical Pharmacy, Faculty of Pharmacy, Universitas Gadjah Mada, Mlati sub- \\ district, Yogyakarta, 5528I, Indonesia, Tel +62 8122792163, Email yasin_nm@ugm.ac.id
}

Background: The determinants of medication adherence in people with diabetes may differ between populations of an area due to social environment, cultural beliefs, socioeconomic conditions, education, and many other factors differences.

Objective: Therefore, this study aims to explore, identify and classify the determinants of medication adherence in several Asian regions.

Methods: A systematic literature review was conducted to gain insight into the determinants of medication adherence. Seven relevant databases (EBSCO, ProQuest, PubMed, ScienceDirect, Scopus, Wiley, dan Taylor and Francis) and hand searching methods were conducted from January 2011 to December 2020. Keywords were compiled based on the PICO method. The selection process used the PRISMA guidelines based on inclusion, and the quality was assessed using Crowe's critical assessment tool. Textual summaries and a conceptual framework model of medication adherence were proposed to aid in the understanding of the factors influencing medication adherence.

Results: Twenty-six articles from countries in several Asian regions were further analyzed. Most studies on type 2 diabetes patients in India used the MMAS-8 scale, and cross-sectional study is the most frequently used research design. The medication adherence rate among diabetic patients was low to moderate. Fifty-one specific factors identified were further categorized into twenty-three subdomains and six domains. Furthermore, the determinants were classified into four categories: inconsistent factors, positively related factors, negatively related factors, and non-associated factors. In most studies, patient-related factors dominate the association with medication adherence. This domain relates to patient-specific demographics, physiological feelings, knowledge, perceptions and beliefs, comorbidities, and other factors related to the patient. Several limitations in this review need to be considered for further research.

Conclusion: Medication adherence to diabetic therapy is a complex phenomenon. Most determinants produced disparate findings in terms of statistical significance. The identified factors can serve various goals related to medication adherence. Policymakers and health care providers should consider patient-related factors.

Keywords: medication adherence, diabetes mellitus, determinants, Asia, patient-related factors, associated factors

\section{Introduction}

In recent decades, the prevalence of diabetes mellitus has increased worldwide and has become a global epidemic. ${ }^{1}$ The highest increase occurred in low-middle income countries in Southeast Asia, South Asia, and West Asia. ${ }^{1}$ Almost $21 \%$ of all diabetes cases worldwide live in the South Asia region; thus, this region is considered the epicenter of the global diabetes epidemic. $^{2}$ Globally, the number of adults with diabetes to increase by more than $50 \%$ over the next 20 years. ${ }^{1}$ This projection is associated with an increasing prevalence of obesity, unhealthy lifestyles, poor eating habits, and prevention efforts from countries in the Asian region. ${ }^{3}$ Two systematic reviews conclude that the inadequate response of the health system to diabetes in several countries in the Asia-Pacific region exacerbates this situation., 
Diabetes requires self-motivation from sufferers to follow a lifelong combination of pharmacological and nonpharmacological therapy; thus, patient adherence to treatment is needed. ${ }^{6}$ The combination seeks to control the risk factors for micro and macrovascular consequences of this disease. ${ }^{6,7}$ Adherence to combination therapy is the key to diabetes treatment but has not received optimal attention from clinicians. ${ }^{8,9}$ Diabetes therapy also depends on the patient's self-management, but therapy failure often occurs due to nonadherent behavior towards treatment. ${ }^{10}$ Such behavior is a significant barrier to the successful treatment of this disease. $^{9,11}$

Research in several developed countries found that the prevalence of medication adherence in diabetes patients ranged from $38.5 \%$ to $93.1 \% .{ }^{12}$ Research in the Middle East region found a lower percentage of medication adherence ranging from $38 \%$ to $41 \% .{ }^{13}$ Non-adherence to medication for diabetes patients in most developed and developing countries shows a high rate. ${ }^{14-16}$ Non-adherence to taking medication in diabetic patients impacts the risk of complications, increasing mortality, increasing the use of health services, increasing treatment costs, decreasing quality of life, and even increasing the country's economic burden. ${ }^{10,17}$ Knowing the determinants of medication adherence could prevent the negative impact. However, these determinant factors have not been optimally identified in diabetic patients in several regions. ${ }^{8}$ This situation reaffirms the need for further investigation of factors that may affect medication adherence in diabetic patients.

Over the past decade, several studies about medications adherence have been conducted in several Asian countries to explore the factors that influence medication adherence among diabetic patients. Several systematic reviews in Europe, Africa, and the Middle East have also identified various factors of medication adherence in diabetic patients. ${ }^{12,13,18}$ However, no systematic review studies have explored its determinants in the Asian regions such as South Asia, Southeast Asia, and East Asia. Even though one of these areas is the epicenter of the global diabetes epidemic. ${ }^{2}$ Most studies on diabetes medication adherence have been conducted in developed countries. However, the social environment, cultural beliefs, socioeconomic conditions, education, and many other factors are very different in developing countries such as the Asian region. ${ }^{19-21}$ Therefore, this study aims to explore, identify and classify the determinants of adherence to diabetes treatment in several Asian areas. These factors can provide information for developing conceptual models used in adherence research and intervention strategies.

\section{Methodology}

\section{Search Strategy}

This review followed the most recent version of the PRISMA (Preferred Reporting Items for Systematic Reviews and Meta-Analyses) guideline. ${ }^{22}$ Seven electronic databases were systematically searched (PubMed, Scopus, ScienceDirect, ProQuest, EBSCO, Wiley Online, and Taylor \& Francis Online) from January 1, 2011, to December 31, $2020 .^{23}$ An initial literature search was performed to find suitable keywords based on the PICO search strategy. ${ }^{24}$ Various terms, words, synonym, and phrases were used to identify relevant papers. A hand search method was also used to address the possibility that studies were not tagged to be missed in the electronic search process. ${ }^{25}$ This method has become a recognized tool in the systematic review process. PRISMA process for abstracts checklist and search query can be found in the supplementary materials (Tables S1 and $\underline{\mathrm{S} 2})$.

\section{Study Selection}

Two research members initially checked the titles for relevance to the study's purpose. Additionally, we evaluated eligibility for inclusion/exclusion based on the abstracts of the research. Full-text papers were reviewed if the abstracts were unclear enough to determine whether an article fulfilled the inclusion criteria. The articles are imported using reference manager software and remove duplicates. Studies identified through the systematic search were eligible for inclusion if they matched the following criteria: 1) The study population was adult patients up to the elderly with type 1 diabetes or type 2 diabetes; 2) the study was conducted at least in one South Asia, East Asia, or Southeast Asia countries; 3) a quantitative or qualitative study on the determinants of medication adherence that investigate at least one factor; 4) The papers were published in English full text, and It will be excluded if the paper is not from the original 
research type. In addition, studies evaluating other than medication adherence were also excluded. Figure 1 illustrates the PRISMA selection procedure.

\section{Study Quality Assessment}

The Crowe Critical Appraisal Tool (CCAT) was used to evaluate the quality of the studies. ${ }^{26}$ CCAT provided validity and reliability data more accurately than informal evaluation methods. It can evaluate both quantitative and qualitative research. ${ }^{26}$ Each item has numerous descriptions that simplify evaluating and rating a category. The CCAT assessment is converted in percentage form and categorized into 4 categories according to the percentage value of each study, they are low (0-39\%); below average (40-59\%); good (60-79\%); very good (80-100\%). ${ }^{26}$ Table 1 summarizes the quality assessment of the articles included.

\section{Data Extraction and Analysis}

The primary outcomes were factors that could influence patients' adherence to medication based on the study's main findings and statistical information. The specific information was collected using an extraction sheet to summarize the results. Two research member extracts the data, and then it is double-checked by another. A multidimensional adherence model was used to classify these factors; (1) patient-related factors, (2) disease-related factors, (3) medication-related factors, (4) healthcare system-related factors, (5) health care provider-related factors, (6) societal-related factors. ${ }^{27,28}$ Based on the extracted data, we created textual summaries and identified the domain of medication adherence factors.

Furthermore, the extracted data were classified into four categories, namely: (1) factors with a positive association (the correlation between these factors increases adherence); (2) factors with a negative association (the correlation

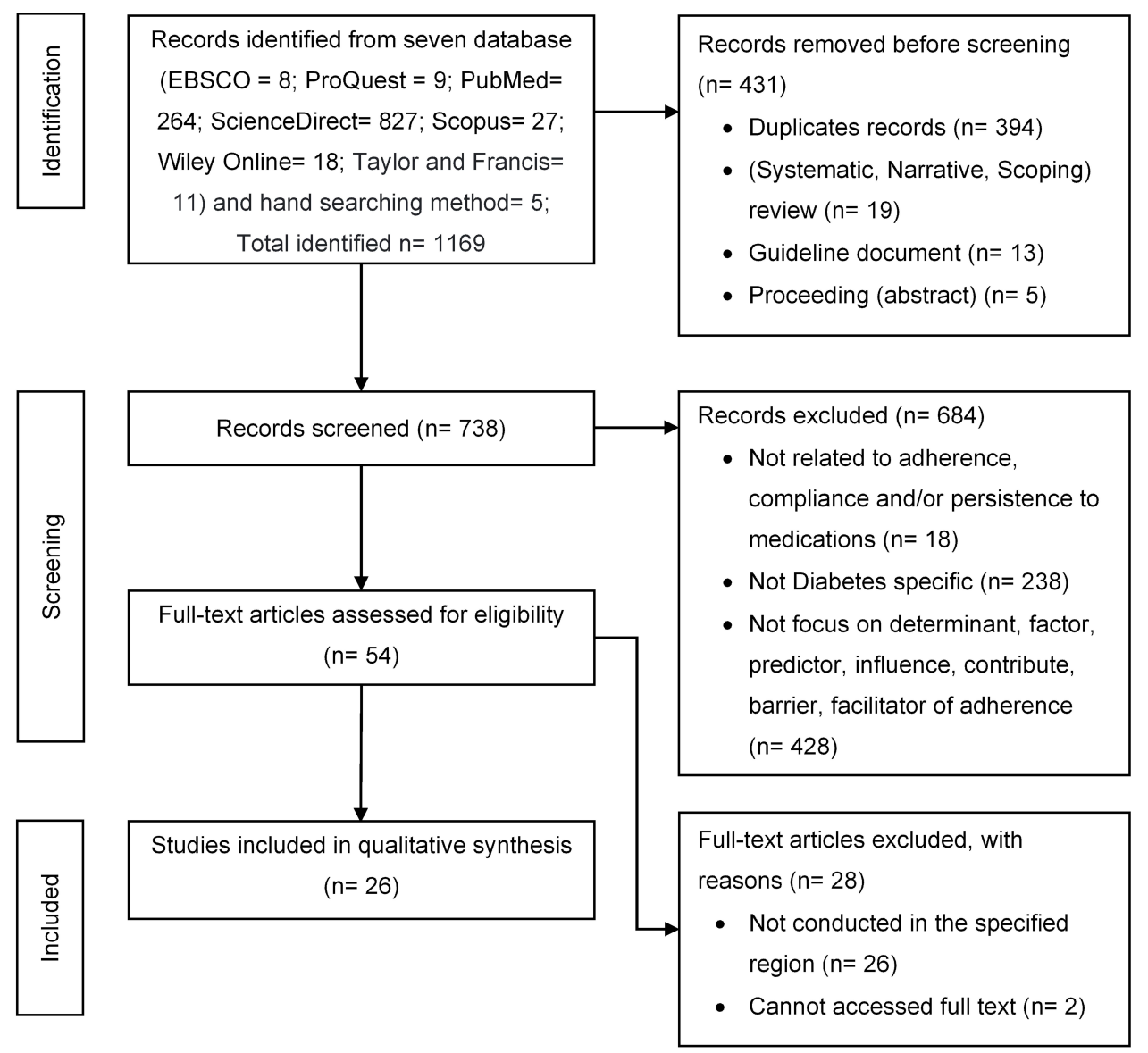

Figure I PRISMA flow diagram for article selection from databases and hand search method.

Note: Page MJ, McKenzie JE, Bossuyt PM, et al. The PRISMA 2020 statement: an updated guideline for reporting systematic reviews. PLoS Med. 202 I; I8(3):el003583. doi:10.1371/journal.pmed.1003583. ${ }^{22}$ 
Table I Characteristics of the Included Studies and Assessment of Their Quality

\begin{tabular}{|c|c|c|c|c|c|c|c|c|}
\hline No & $\begin{array}{l}\text { Author/Country/Setting/Design/TYPE of } \\
\text { DM }\end{array}$ & $\begin{array}{l}\text { Sample } \\
\text { Size }\end{array}$ & $\begin{array}{l}\text { Adherence } \\
\text { Instrument }\end{array}$ & Adherence Cut Point & $\begin{array}{l}\text { Adherence } \\
\text { Rate (\%) }\end{array}$ & $\begin{array}{l}\text { Determinants Found } \\
\text { Significant to Adherence }\end{array}$ & $\begin{array}{l}\text { Determinants Found } \\
\text { Not Significant to } \\
\text { Adherence }\end{array}$ & $\begin{array}{l}\text { CCAT } \\
\text { Score } \\
\text { (\%) }\end{array}$ \\
\hline I. & $\begin{array}{l}\text { (Al-Qazaz et al, } 201 \mathrm{I} \text { )/Malaysia/Hospital/Cross- } \\
\text { sectional/T2DM. }{ }^{42}\end{array}$ & 540 & MMAS-8 & $\begin{array}{l}-<6 \text { points (low adherence) } \\
-6 \text { to }<8 \text { (medium adherence) } \\
-\leq 8 \text { (high adherence) }\end{array}$ & $\begin{array}{l}\text { - Low adherence } \\
\text { (79.2\%) } \\
\text { - High adherence } \\
(20.8 \%)\end{array}$ & $\begin{array}{l}\text { - Education level } \\
\text { - Medication number } \\
\text { - Diabetes knowledge }\end{array}$ & $\begin{array}{l}\text { - Age } \\
\text { - Gender } \\
\text { - Ethnicity } \\
\text { - Diabetes duration }\end{array}$ & 81 \\
\hline 2. & $\begin{array}{l}\text { (Chew et al, 2015)/Malaysia/Public Health } \\
\text { Clinic/Cross-sectional/T2DM. }{ }^{44}\end{array}$ & 668 & MMAS-8 & $\begin{array}{l}\text { - }<6 \text { points (low adherence) } \\
-6 \text { to }<8 \text { (medium adherence) } \\
-\leq 8 \text { (high adherence) }\end{array}$ & $\begin{array}{l}\text { - Low adherence } \\
(43 \%) \\
\text { - Medium to high } \\
\text { adherence (57\%) }\end{array}$ & $\begin{array}{l}\text { - Age } \\
\text { - Income } \\
\text { - Depressive symptoms }\end{array}$ & $\begin{array}{l}\text { - Ethnicity } \\
\text { - Exercise } \\
\text { - Quality of Life } \\
\text { - Type of medicine } \\
\text { - Occupation }\end{array}$ & 82 \\
\hline 3. & $\begin{array}{l}\text { (Nazir et al, 2015)/Pakistan/Hospital/Cross- } \\
\text { sectional/T2DM. }{ }^{47}\end{array}$ & 392 & MMAS-U & $\begin{array}{l}\text { - } 0 \text { to } \leq 6 \text { (low adherence) } \\
\text { - } 6 \text { to } 7 \text { (medium adherence) } \\
\text { - } 8 \text { (high adherence) }\end{array}$ & $\begin{array}{l}\text { - Low adherence } \\
(71.94 \%) \\
\text { - Medium to high } \\
\text { adherence } \\
(28.06 \%)\end{array}$ & - & $\begin{array}{l}\text { - Age } \\
\text { - Gender } \\
\text { - Marital Status } \\
\text { - Education } \\
\text { - Income } \\
\text { - Locality } \\
\text { - Occupation } \\
\text { - Duration of disease } \\
\text { - Diabetes-related } \\
\text { knowledge }\end{array}$ & 79 \\
\hline 4. & $\begin{array}{l}\text { (Sankar et al, 2015)//ndia/Community-dwelling } \\
\text { /Cross-sectional/TIDM and T2DM. }{ }^{31}\end{array}$ & 346 & MMAS-8 & $\begin{array}{l}-<6 \text { (Poor adherence) } \\
\bullet \geq 6 \text { (Good adherence) }\end{array}$ & $\begin{array}{l}\text { - Poor adherence } \\
(73.9 \%) \\
\text { - Good } \\
\text { adherence (26\%) }\end{array}$ & $\begin{array}{l}\text { - Lower economic status } \\
\text { - Irregular monitoring of blood } \\
\text { sugar } \\
\text { - Inadequate instructions from } \\
\text { health care } \\
\text { - Management of Diabetes } \\
\text { - Lack of family support. }\end{array}$ & - & 83 \\
\hline 5. & $\begin{array}{l}\text { (Zhang et al, 2015)/China/Hospital and } \\
\text { community clinic/Cross-sectional/T2DM. }{ }^{40}\end{array}$ & 2538 & MMAS-4 & $\begin{array}{l}\text { - } 0 \text { to I (low adherence) } \\
\text { - 2-3 (intermediate adherence) } \\
\text { - } 4 \text { (high adherence) }\end{array}$ & $\begin{array}{l}\text { - Low adherence } \\
(7.1 \%) \\
\text { - Intermediate } \\
\text { adherence } \\
(33.9 \%) \\
\text { - High adherence } \\
(59 \%)\end{array}$ & $\begin{array}{l}\text { - Age } \\
\text { - Education level } \\
\text { - Poor education, } \\
\text { - Smoking status } \\
\text { - High Body Mass Index, } \\
\text { - Use Of Insulin, } \\
\text { - Depression }\end{array}$ & $\begin{array}{l}\text { - HbAlc level } \\
\text { - Gender } \\
\text { - Duration of diabetes } \\
\text { - Education level } \\
\text { - Occupation } \\
\text { - Family history of } \\
\text { diabetes }\end{array}$ & 78 \\
\hline
\end{tabular}




\begin{tabular}{|c|c|c|c|c|c|c|c|c|}
\hline 6. & 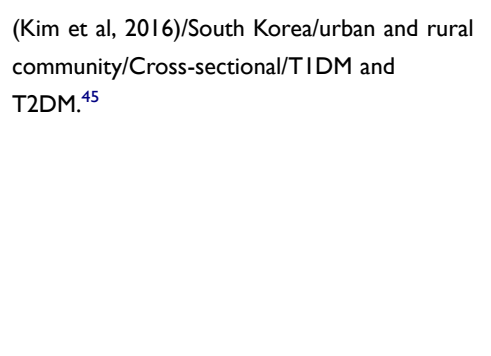 & 741 & $\begin{array}{l}\text { Unvalidated } \\
\text { questionnaire }\end{array}$ & $\begin{array}{l}\text { - Patients did not report taking anti- } \\
\text { diabetic medications. (non-acherent) } \\
\text { - Patients reported taking anti-diabetic } \\
\text { medications (acherent) }\end{array}$ & $\begin{array}{l}\text { - Non-adherence } \\
(55.7 \%) \\
\text { - Adherence } \\
(44.3 \%)\end{array}$ & $\begin{array}{l}\text { - Home location (urban or } \\
\text { rural) } \\
\text { - Age } \\
\text { - Gender } \\
\text { - Alcohol status } \\
\text { - High blood pressure } \\
\text { - High total cholesterol } \\
\text { - Lack of family history of } \\
\text { diabetes }\end{array}$ & $\begin{array}{l}\text { - Incomes } \\
\text { - Education levels } \\
\text { - Smoking status } \\
\text { - Exercise } \\
\text { - Marital status } \\
\text { - Occupation }\end{array}$ & 70 \\
\hline 7. & $\begin{array}{l}\text { (Wu and Liu, 2016)/China/Hospital/Cross- } \\
\text { sectional/T2DM.3. }\end{array}$ & 130 & MMAS-8 & $\begin{array}{l}-0 \text { to } \leq 6 \text { (poor adherence) } \\
-6 \text { to } 8 \text { (optimal adherence) }\end{array}$ & $\begin{array}{l}\text { - Poor adherence } \\
\text { (54.6\%) } \\
\text { - Optimal } \\
\text { adherence } \\
(45.4 \%)\end{array}$ & $\begin{array}{l}\text { Facilitating factors } \\
\text { - Having the drug on hand } \\
\text { Barriers factors } \\
\text { - Being away from home or } \\
\text { eating out } \\
\text { - Not accepting the disease } \\
\text { - Ignorance of life-long drug } \\
\text { adherence } \\
\text { - When busy } \\
\text { - Poor memory }\end{array}$ & $\begin{array}{l}\text { - Age } \\
\text { - Sex } \\
\text { - Marital status } \\
\text { - Duration of disease } \\
\text { - Education level } \\
\text { - Monthly income } \\
\text { - Insurance } \\
\text { - Number of medications } \\
\text { - Frequency of medication }\end{array}$ & 74 \\
\hline 8. & (Gu et al, 2017)/China/Hospital/NA/T2DM. ${ }^{37}$ & 331 & MMAS-8-CN & $\begin{array}{l}-<6 \text { points (low adherence) } \\
-6 \text { to } \leq 8 \text { (high adherence) }\end{array}$ & $\begin{array}{l}\text { - Low adherence } \\
(72.8 \%) \\
\text { • High adherence } \\
(27.1 \%)\end{array}$ & $\begin{array}{l}\text { - Social support } \\
\text { - Reimbursement }\end{array}$ & $\begin{array}{l}\text { - Sex } \\
\text { - Age } \\
\text { - Marital status } \\
\text { - Family income } \\
\text { - Education level } \\
\text { - Employment status } \\
\text { - Insurance type } \\
\text { - BMI } \\
\text { - Duration of disease }\end{array}$ & 72 \\
\hline 9. & $\begin{array}{l}\text { (Islam et al, 2017)/Bangladesh/Hospital/ } \\
\text { Qualitative study/T2DM. }{ }^{52}\end{array}$ & 12 & HbAlc level & $\begin{array}{l}- \text { HbAIc } \geq 7 \text { (uncontrolled) } \\
\text { - HbAIc }<7 \text { (controlled) }\end{array}$ & $\begin{array}{l}- \text { Uncontrolled } \\
(75 \%) \\
\text { - Controlled } \\
(25 \%)\end{array}$ & $\begin{array}{l}\text { - Education } \\
\text { - Forgetfulness } \\
\text { - Cost of medications } \\
\text { - Self-management } \\
\text { - Knowledge } \\
\text { - Psychological stress }\end{array}$ & - & 82 \\
\hline
\end{tabular}


Table I (Continued).

\begin{tabular}{|c|c|c|c|c|c|c|c|c|}
\hline No & $\begin{array}{l}\text { Author/Country/Setting/Design/TYPE of } \\
\text { DM }\end{array}$ & $\begin{array}{l}\text { Sample } \\
\text { Size }\end{array}$ & $\begin{array}{l}\text { Adherence } \\
\text { Instrument }\end{array}$ & Adherence Cut Point & $\begin{array}{l}\text { Adherence } \\
\text { Rate }(\%)\end{array}$ & $\begin{array}{l}\text { Determinants Found } \\
\text { Significant to Adherence }\end{array}$ & $\begin{array}{l}\text { Determinants Found } \\
\text { Not Significant to } \\
\text { Adherence }\end{array}$ & $\begin{array}{l}\text { CCAT } \\
\text { Score } \\
(\%)\end{array}$ \\
\hline 10. & $\begin{array}{l}\text { (Lee et al, 2017)/Singapore/Primary care } \\
\text { outpatient clinic/Cross-sectional/T2DM. }{ }^{16}\end{array}$ & 382 & MARS-5 & $\begin{array}{l}\bullet \leq 25 \text { (low adherence) } \\
\bullet>25 \text { (high adherence) }\end{array}$ & $\begin{array}{l}\text { - Low adherence } \\
\text { (57.1\%) } \\
\text { - High adherence } \\
(42.9 \%)\end{array}$ & $\begin{array}{l}\text { - Age } \\
\text { - Ethnicity } \\
\text { - HbAIc }\end{array}$ & $\begin{array}{l}\text { - Marital status } \\
\text { - Administration of } \\
\text { medication } \\
\text { - Total number of daily/ } \\
\text { regular medications }\end{array}$ & 82 \\
\hline 11. & $\begin{array}{l}\text { (Mentock et al, 2017)/India/Diabetic clinic/ } \\
\text { Cross-sectional/T2DM. } .^{30}\end{array}$ & 204 & $\begin{array}{l}\text { Blood glucose } \\
\text { level }\end{array}$ & $\begin{array}{l}\text { - Fasting blood glucose } 130 \mathrm{mg} / \mathrm{dl} \\
\text { (uncontrolled) } \\
\text { - } 70-130 \mathrm{mg} / \mathrm{dl} \text { (controlled) }\end{array}$ & $\begin{array}{l}- \text { Uncontrolled } \\
(62.2 \%) \\
\text { - Controlled } \\
(37.7 \%)\end{array}$ & $\begin{array}{l}\text { - Age } \\
\text { - Home location } \\
\text { - High treatment costs }\end{array}$ & $\begin{array}{l}\text { - Gender } \\
\text { - Household income } \\
\text { - Religion } \\
\text { - Marital status } \\
\text { - Diabetic diet } \\
\text { - Transportation } \\
\text { - Social support } \\
\text { - Health care provider } \\
\text { - Medication availability }\end{array}$ & 80 \\
\hline 12. & $\begin{array}{l}\text { (Abdullah et al, 2019)/Malaysia/Hospital/Cross- } \\
\text { sectional/T2DM. }{ }^{41}\end{array}$ & 232 & MCQ & $\begin{array}{l}\bullet<27 \text { (non-adherence) } \\
\bullet \geq 27 \text { (adherence) }\end{array}$ & $\begin{array}{l}\text { - Non-adherence } \\
(44.8 \%) \\
\text { - Adherence } \\
(55.2 \%)\end{array}$ & $\begin{array}{l}\text { - Ethnicity } \\
\text { - Glycemic levels/HbAIc }\end{array}$ & $\begin{array}{l}\text { - Gender } \\
\text { - Marital status } \\
\text { - Education levels } \\
\text { - Income } \\
\text { - Occupation } \\
\text { - Duration of disease } \\
\text { - Comorbidities } \\
\text { - Type of treatment } \\
\text { - Number of drugs }\end{array}$ & 82 \\
\hline 13. & $\begin{array}{l}\text { (Balasubramaniam et al, 2019)/Malaysia/ } \\
\text { Hospital/Cross-sectional/T2DM. }{ }^{43}\end{array}$ & 384 & MMAS-8 & $\begin{array}{l}-<6 \text { points (low adherence) } \\
\bullet 6 \text { to }<8 \text { (medium adherence) } \\
-\leq 8 \text { (high adherence) }\end{array}$ & $\begin{array}{l}\text { - Low adherence } \\
(39.6 \%) \\
\text { - Medium } \\
\text { adherence } \\
(36.2 \%) \\
\text { - High adherence } \\
(23.7 \%)\end{array}$ & $\begin{array}{l}\text { - Illness Coherence } \\
\text { - Identity } \\
\text { - Consequences } \\
\text { - Emotional Representations } \\
\text { - Timeline Cyclical }\end{array}$ & $\begin{array}{l}\text { - Age } \\
\text { - Gender } \\
\text { - Ethnicity } \\
\text { - Duration of disease } \\
\text { - BMI } \\
\text { - Educational level } \\
\text { - Marital Status } \\
\text { - Occupation } \\
\text { - Comorbidities } \\
\text { - Timeline Acute/Chronic } \\
\text { - Personal Control } \\
\text { - Treatment Control }\end{array}$ & 85 \\
\hline
\end{tabular}




\begin{tabular}{|c|c|c|c|c|c|c|c|c|}
\hline 14. & $\begin{array}{l}\text { (Horii et al, 2019)/Japan/NA/Observational } \\
\text { retrospective study/T2DM. }{ }^{51}\end{array}$ & 884 & PDC & $\begin{array}{l}-P D C \text { index }<0.8 \text { (non-adherence) } \\
-P D C \text { index } \geq 0.8 \text { (adherence) }\end{array}$ & $\begin{array}{l}\text { - Non-adherence } \\
(50.2 \%) \\
\text { - Adherence } \\
(49.7 \%)\end{array}$ & $\begin{array}{l}\text { - Age } \\
\text { - Number of medication } \\
\text { - Regular visits }\end{array}$ & - & 81 \\
\hline 15. & $\begin{array}{l}\text { (Nonogaki et al, 2019) Cambodia/Patient } \\
\text { Information Centre/Cross-sectional/TIDM and } \\
\text { T2DM. }{ }^{46}\end{array}$ & 773 & MMAS-4 & $\begin{array}{l}\text { - } 3 \text { to } 4 \text { (low adherence) } \\
\text { - I to } 2 \text { (medium adherence) } \\
\text { - } 0 \text { (high adherence) }\end{array}$ & $\begin{array}{l}\text { - Low to Medium } \\
\text { adherence } \\
(50.7 \%) \\
\text { - High adherence } \\
(49.3 \%)\end{array}$ & $\begin{array}{l}\text { - Higher monthly family income } \\
\text { - Regular follow-ups at health } \\
\text { facilities } \\
\text { - Community-based peer } \\
\text { educator group } \\
\text { - Alcohol status } \\
\text { - Following a special diet for } \\
\text { diabetes mellitus } \\
\text { - Complications }\end{array}$ & - & 83 \\
\hline 16. & $\begin{array}{l}\text { (Rathish et al, 2019)/Sri Lanka/Hospital/Cross- } \\
\text { sectional/T2DM. }{ }^{74}\end{array}$ & 350 & MGL & $\begin{array}{l}\text { - } 0 \text { to I (low adherence) } \\
-2 \text { to } 4 \text { (high to moderate adherence) }\end{array}$ & $\begin{array}{l}\text { - Low adherence } \\
(7.5 \%) \\
\text { - Moderate and } \\
\text { high adherence } \\
(92.5 \%)\end{array}$ & - & - Health Insurance type & 58 \\
\hline 17. & $\begin{array}{l}\text { (Aditama et al, 2020)/Indonesia/primary health } \\
\text { care/Qualitative Explanatory sequential/ } \\
\text { T2DM. }{ }^{2}\end{array}$ & 40 & $\begin{array}{l}\text { Adherence } \\
\text { behavior } \\
\text { questionnaire }\end{array}$ & $\begin{array}{l}\text { - Mean scores of }<\text { I (non-adherence) } \\
\text { - Mean scores of I (Adherence) }\end{array}$ & $\begin{array}{l}\text { - Non-adherence } \\
(80 \%) \\
\text { - Adherence } \\
(20 \%)\end{array}$ & $\begin{array}{l}\text { - Forgetfulness } \\
\text { - Not to take the medication } \\
\text { - Non-availability of the Drug } \\
\text { product }\end{array}$ & & 59 \\
\hline 18. & $\begin{array}{l}\text { (Hussain et al, 2020)/Pakistan/Hospital/Cross- } \\
\text { sectional/T2DM. }\end{array}$ & 524 & BARS & $\begin{array}{l}\bullet<7 \text { (rarely) } \\
-8 \text { to } 13 \text { (often) } \\
\bullet 14 \text { to } 20 \text { (frequently) } \\
->20 \text { (mostly) }\end{array}$ & $\begin{array}{l}\text { - Rarely (4\%) } \\
\text { - Often (73\%) } \\
\text { - Frequently } \\
\text { (16\%) } \\
\text { - Mostly (5\%) }\end{array}$ & $\begin{array}{l}\text { - Inadequate health literacy } \\
\text { - Skipping medication doses } \\
\text { - Altering medication doses } \\
\text { without health professional } \\
\text { consultation }\end{array}$ & - & 81 \\
\hline 19. & $\begin{array}{l}\text { (Wulandari et al, 2020)/Indonesia//community } \\
\text { health centre/Cross-sectional/T2DM. }{ }^{58}\end{array}$ & 143 & HbAIc level & $\begin{array}{l}- \text { HbAlc } \geq 7 \text { (Low adherence) } \\
- \text { HbAlc }<7 \text { (Adherence) }\end{array}$ & $\begin{array}{l}\text { - Low adherence } \\
(75.5 \%) \\
\text { - Adherence } \\
(24.5 \%)\end{array}$ & - Disease duration & $\begin{array}{l}\text { - Age } \\
\text { - Gender } \\
\text { - Level of education } \\
\text { - Occupation } \\
\text { - Other chronic diseases } \\
\text { - Number of medicine } \\
\text { - Number of regular daily } \\
\text { drugs }\end{array}$ & 77 \\
\hline
\end{tabular}

(Continued) 
Table I (Continued).

\begin{tabular}{|c|c|c|c|c|c|c|c|c|}
\hline No & $\begin{array}{l}\text { Author/Country/Setting/Design/TYPE of } \\
\text { DM }\end{array}$ & $\begin{array}{l}\text { Sample } \\
\text { Size }\end{array}$ & $\begin{array}{l}\text { Adherence } \\
\text { Instrument }\end{array}$ & Adherence Cut Point & $\begin{array}{l}\text { Adherence } \\
\text { Rate (\%) }\end{array}$ & $\begin{array}{l}\text { Determinants Found } \\
\text { Significant to Adherence }\end{array}$ & $\begin{array}{l}\text { Determinants Found } \\
\text { Not Significant to } \\
\text { Adherence }\end{array}$ & $\begin{array}{l}\text { CCAT } \\
\text { Score } \\
(\%)\end{array}$ \\
\hline 20. & $\begin{array}{l}\text { (Xu et al, 2020)/China/Elderly clinic/Cross- } \\
\text { sectional/TIDM and T2DM. }{ }^{39}\end{array}$ & 1002 & MGL & $\begin{array}{l}\text { - } 0 \text { to I (Non-adherence) } \\
\text { - } 2 \text { to } 4 \text { (adherence) }\end{array}$ & $\begin{array}{l}\text { - Non-adherence } \\
(19.9 \%) \\
\text { - Adherence } \\
(80.1 \%)\end{array}$ & $\begin{array}{l}\text { - Gender } \\
\text { - Disease duration } \\
\text { - Perceived importance of } \\
\text { medication adherence }\end{array}$ & $\begin{array}{l}\text { - Residence } \\
\text { - Age } \\
\text { - Education Level } \\
\text { - Marital Status } \\
\text { - Employment Status } \\
\text { - Personal Annual Income } \\
\text { - Health Insurance } \\
\text { - Complication } \\
\text { - Type Of Medication } \\
\text { - Mental Health Status }\end{array}$ & 75 \\
\hline 21. & $\begin{array}{l}\text { (Zhang et al, 2020)/Singapore/Primary care } \\
\text { polyclinics/Cross-sectional/T2DM. }{ }^{50}\end{array}$ & 448 & MGL & $\begin{array}{l}\text { - } 0 \text { to I (low adherence) } \\
\text { - } 2 \text { to } 3 \text { (moderate adherence) } \\
\text { - } 4 \text { (high adherence) }\end{array}$ & $\begin{array}{l}\text { - Low adherence } \\
(59.8 \%) \\
\text { - Moderate to } \\
\text { high adherence } \\
(40.1 \%)\end{array}$ & $\begin{array}{l}\text { - Age } \\
\text { - HbAlc level } \\
\text { - Home glucose monitoring } \\
\text { - Peripheral Neuropathy }\end{array}$ & $\begin{array}{l}\text { - Ethnicity } \\
\text { - Education } \\
\text { - Marital status } \\
\text { - Income } \\
\text { - Household type } \\
\text { - Occupation } \\
\text { - Payment for medical bills } \\
\text { - Duration of diabetes } \\
\text { - Diabetic medications } \\
\text { - Comorbidities } \\
\text { - Complications } \\
\text { - Social support }\end{array}$ & 80 \\
\hline 22. & $\begin{array}{l}\text { (Basu et al, 2015)/India/Hospital/Cross- } \\
\text { sectional/T2DM. }{ }^{32}\end{array}$ & 385 & MMAS-8 & $\begin{array}{l}-<6 \text { points (Poor adherence) } \\
-6 \text { to }<8 \text { (medium adherence) } \\
-\leq 8 \text { (Good adherence) }\end{array}$ & $\begin{array}{l}\text { - Poor adherence } \\
(25.5 \%) \\
\text { - Good } \\
\text { adherence } \\
(74.5 \%)\end{array}$ & $\begin{array}{l}\text { - Socioeconomic status } \\
\text { - Medication type } \\
\text { - Skip doses on exhausting drug } \\
\text { stocks }\end{array}$ & $\begin{array}{l}\text { - Age } \\
\text { - Gender } \\
\text { - Spouse } \\
\text { - Education } \\
\text { - Diabetes duration } \\
\text { - BMI }\end{array}$ & 81 \\
\hline 23. & $\begin{array}{l}\text { (Mukherjee, } 2013 \text { )/India/Hospital/Cross- } \\
\text { sectional/T2DM. }{ }^{35}\end{array}$ & 470 & HbAIC level & $\begin{array}{l}\text { - HbAlc } \geq 7 \text { (non-compliance) } \\
\text { - HbAlc }<7 \text { (Compliance) }\end{array}$ & $\begin{array}{l}\text { - Non- } \\
\text { compliance } \\
(57.7 \%) \\
\text { - Compliance } \\
(42.3 \%)\end{array}$ & $\begin{array}{l}\text { - Age } \\
\text { - Sex } \\
\text { - Education status } \\
\text { - Income } \\
\text { - Duration of diabetes } \\
\text { - Type of medication } \\
\text { - Knowledge on complications } \\
\text { of diabetes }\end{array}$ & $\begin{array}{l}\text { - Marital status } \\
\text { - Followed diet plan }\end{array}$ & 68 \\
\hline
\end{tabular}




\begin{tabular}{|c|c|c|c|c|c|c|c|c|}
\hline 24. & $\begin{array}{l}\text { (Thapar et al, 2020)/India/Hospital/Cross- } \\
\text { sectional/T2DM..34 }\end{array}$ & 124 & MMAS-8 & $\begin{array}{l}-<6 \text { points (low adherence) } \\
-6 \text { to }<8 \text { (medium adherence) } \\
-\leq 8 \text { (high adherence) }\end{array}$ & $\begin{array}{l}\text { - Low adherence } \\
(43.5 \%) \\
\text { - Medium } \\
\text { adherence (29\%) } \\
\text { - High adherence } \\
(27.4 \%)\end{array}$ & - Absence of Side effects & $\begin{array}{l}\text { - Age } \\
\text { - Gender } \\
\text { - Marital status } \\
\text { - Socioeconomic status } \\
\text { - Regular blood } \\
\text { monitoring } \\
\text { - Family history of } \\
\text { diabetes } \\
\text { - Presence of } \\
\text { comorbidities } \\
\text { - Smoking status } \\
\text { - Alcohol status } \\
\text { - Distance from hospital } \\
\text { - Cost of drugs } \\
\text { - Means of conveyance } \\
\text { - Number of medications } \\
\text { - Insulin injection } \\
\text { - Duration of diabetes } \\
\text { - Diabetic complications } \\
\text { - Fasting Blood Glucose } \\
\text { Level }\end{array}$ & 83 \\
\hline 25. & $\begin{array}{l}\text { (Haghighatpanah et al, 2018)/India/ } \\
\text { Retrospective observational/T2DM. }\end{array}$ & 657 & HbAIC level & $\begin{array}{l}- \text { HbAlc } \geq 7 \text { (Poor glycemic control) } \\
- \text { HbAIc }<7 \text { (Good glycemic control) }\end{array}$ & $\begin{array}{l}\text { - Poor glycemic } \\
\text { control (78.2\%) } \\
\text { - Good glycemic } \\
\text { control (27.1\%) }\end{array}$ & $\begin{array}{l}\text { - Gender } \\
\text { - Age } \\
\text { - BMI } \\
\text { - Occupation } \\
\text { - Medical history } \\
\text { - Triglyceride level } \\
\text { - High-density lipoprotein level } \\
\text { - Duration of diabetes } \\
\text { - Type of medication } \\
\text { - Presence of comorbidity }\end{array}$ & $\begin{array}{l}\text { - Alcohol status } \\
\text { - Smoking status } \\
\text { - Types of payment } \\
\text { - Family history } \\
\text { - Number of complication }\end{array}$ & 81 \\
\hline 26. & $\begin{array}{l}\text { (Basu et al, 2018)/India/Cross-sectional } \\
\text { /T2DM. }{ }^{36}\end{array}$ & 375 & SDSCA & $\begin{array}{l}\text { - Missing anti-diabetic- medications } \\
\text { more than I-d in previous } 7 \mathrm{~d} \text { (Non- } \\
\text { adherent) } \\
\text { - Missing anti-diabetic- medications at } \\
\text { most I-d in previous } 7 \mathrm{~d} \text { (Adherent) }\end{array}$ & $\begin{array}{l}\text { - Non-adherent } \\
(17.6 \%) \\
\text { - Adherent } \\
(82.4 \%)\end{array}$ & $\begin{array}{l}\text { - Education < } 5 \mathrm{yr} \\
\text { - Absence of hypertension } \\
\text { comorbidity }\end{array}$ & $\begin{array}{l}\text { - Living in the nuclear } \\
\text { family } \\
\text { - Insulin therapy } \\
\text { - Family assistance for } \\
\text { taking medication }\end{array}$ & 66 \\
\hline
\end{tabular}

Abbreviations: TIDM, Type I Diabetes Mellitus; T2DM, Type 2 Diabetes Mellitus; MMAS-8, Morisky Medication Adherence Scale-8; MMAS-U, The Urdu version of Morisky Medication Adherence Scale; MMAS-4, Morisky Medication Adherence Scale-4; MMAS-8-CN, The Chinese version of the Morisky Medication Adherence Scale; HbAIc, Glycated Hemoglobin; MARS-5, the five-question Medication Adherence Report Scale; MCQ, Medication Compliance Questionnaire; PDC, Proportion of Days Covered; MGL, Morisky, Green, and Levine adherence scale; BARS, Brief Adherence Rating Scale; SDSCA, Summary of Diabetes Self-care Activities; BMI, Body Mass Index. 


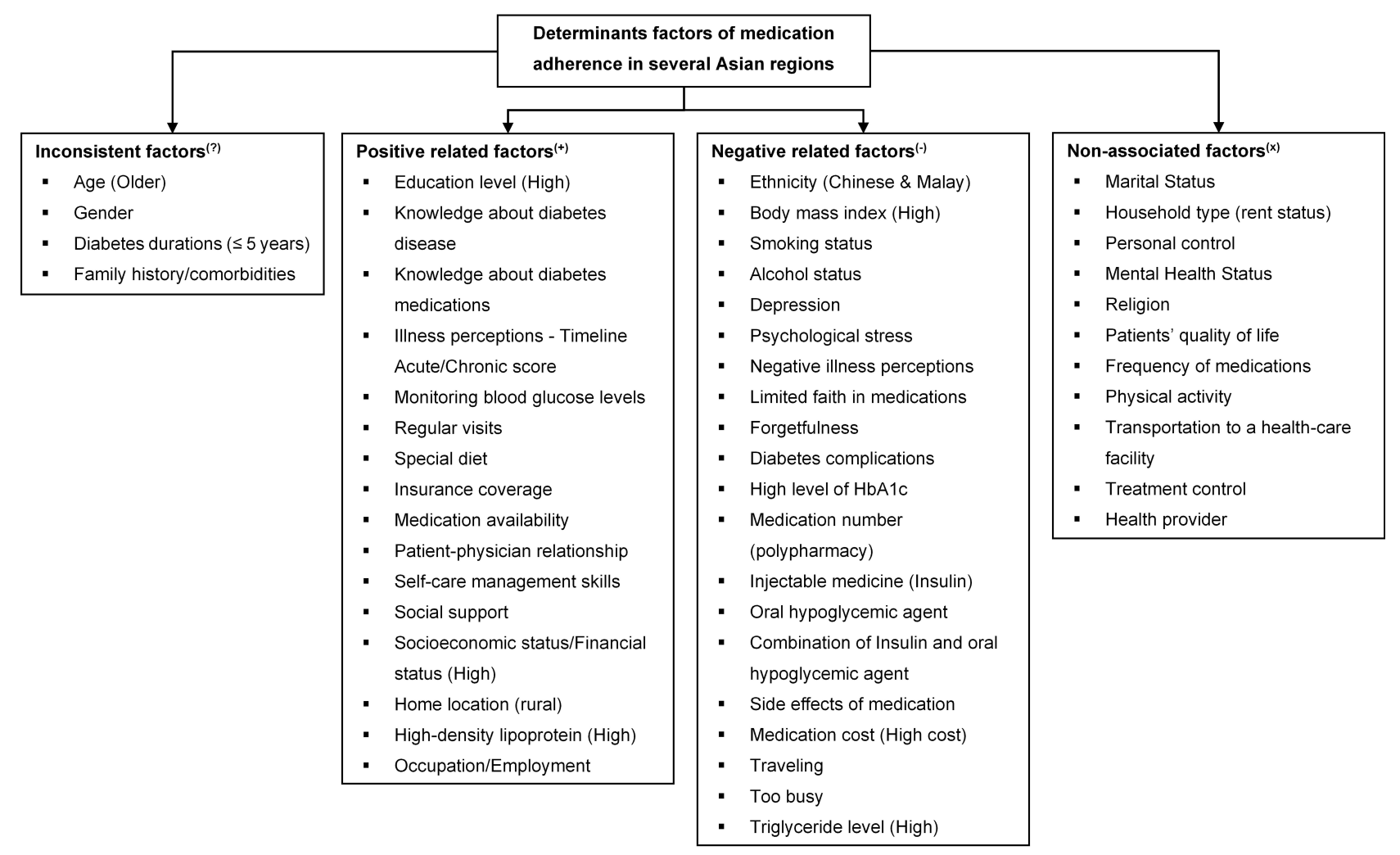

Figure 2 Determinants of medication adherence in several Asian regions.

Notes: ${ }^{(?)}$ Inconsistent association, the association of these factors is inconsistent with diabetes medication adherence. ${ }^{(+)}$Positive association, the association of these factors enhances or facilitates diabetes medication adherence. ${ }^{(-)}$Negative association, the association of these factors reduces or barriers to diabetes medication adherence. ${ }^{(\times)}$Nonassociated factors, these factors are not associated with medication adherence.

between these factors decreases adherence), (3) factors with no association; (4) inconsistent associated factors (Figure 2). A conceptual framework model of medication adherence was proposed to aid in the understanding of the factors influencing medication adherence in Asian diabetic patients. We did not perform a meta-analysis due to study designs, medication adherence scale, sampling procedures, and population variability. Heterogeneity in essential aspects of the chosen study methodology prevented us from conducting a meta-analysis. If studies are too heterogeneous to compare, meta-analysis should be avoided, as the results may be meaningless. ${ }^{29}$ Discussions with other research members handled disagreements in assessing a paper from all these systematic review processes.

\section{Results}

The electronic literature search showed 1164 articles from seven databases and five from hand search methods, bringing 1169 articles found. However, 431 articles were excluded at the identification stage due to duplication and the type of articles not meeting the inclusion criteria. The second screening excluded 684 papers because they were not associated with medication adherence factors in diabetic patients. Furthermore, 28 inaccessible full-text papers and different country regions are also excluded. Finally, we identified 26 papers that fulfilled the inclusion criteria (Figure 1). A cross-sectional study is the most frequently used research design in articles about medication adherence in diabetes patients in many Asian regions $(\mathrm{n}=21)$. Most studies on medication adherence factors in diabetic patients were conducted on type 2 diabetes patients in India ${ }^{30-36}(n=7)$, China ${ }^{37-40}(n=4)$ and Malaysia ${ }^{41-44}(n=4)$. Meanwhile, other studies were conducted in nine countries from three Asian regions. Four research involved both type 1 and type 2 diabetes patients, and none of the investigations were done among type 1 diabetic patients. ${ }^{31,39,45,46}$ Based on the CCAT score, most of the listed studies are of good quality.

The indirect method using various self-reported questionnaires was the primary strategy to identify factors associated with medication adherence $(\mathrm{n}=18)$. The MMAS-based self-reported questionnaire is the common scale used in this 
review $(\mathrm{n}=11) .{ }^{31,32,34,37,38,40,42-44,46,47}$ These results are also found in most studies measuring chronic disease medication adherence because it is practical for a large population. ${ }^{48,49}$ Overall, the average medication adherence among diabetic patients was low to moderate, with an average adherence rate of $20 \%$ to $92.5 \%$. The definition of medication adherence in each study was not explicitly described. It is challenging to be generalized because of the various types of adherence scales used. Adherence rates in most studies used the cut-off point of a self-reported questionnaire $(\mathrm{n}=18)$, an unvalidated questionnaire $(n=2)$, proportion days covered $(n=1)$, or the percentage cut-off point for blood glucose biological marker $(\mathrm{HbAlc})(\mathrm{n}=4)$.

Fifty-one factors reviewed were categorized into six domains and twenty-three subdomains from the included studies. Twenty specific factors are negatively associated with medication adherence $(n=20)$. Sixteen specific factors were positively associated with medication adherence $(n=16)$. In addition, this review has identified four inconsistent factors with adherence $(n=4)$ and eleven factors not associated with medication adherence $(n=11)$. Detailed information about the papers included in this review, including research design, sample size, method of assessing adherence, adherence rate, primary findings, and research quality assessment, are summarized in Table 1.

\section{Patient-Related Factors}

The findings of all factors in the patient-related factor domain have been grouped into seven sub-domains (demographics specific, knowledge, comorbidities, psychological feelings, perceptions and beliefs, quality of life, and additional factors). Four factors inconsistent with medication adherence in the specific demographic sub-domain are older age, gender, diabetes duration ( $\leq 5$ years), and family history/comorbidities. These factors were positively associated in several studies but also found to have a negative or no association at all in other studies. For example, older age in Korea and India is associated with poor medication adherence. ${ }^{35,45}$ However, studies in Malaysia, China, Singapore, Japan, and India found that older diabetic patients were found to have better medication adherence. ${ }^{16,30,33,40,44,50,51}$ Another example in one study in India stated that diabetic patients with comorbid hypertension were more obedient to their diabetes treatment. ${ }^{36}$ These results differ from two other studies examining this factor on medication adherence. ${ }^{33,45}$ The possible reason is the patient's perception of susceptibility to disease and the perceived benefit of the drug taken. ${ }^{36}$

Most of the specific factors in this domain were negatively associated with medication adherence. Eleven of the 25 specific factors were negatively associated, and six positively related to medication adherence. They are education level, ${ }^{35,36,40,42,52}$ socioeconomic status/Financial status, ${ }^{31,32,35,44,46}$ home location, ${ }^{30,45}$ knowledge of diabetes, ${ }^{35,42,52}$ knowledge about diabetes medications, ${ }^{53}$ and illness perceptions. ${ }^{43}$ Five studies in Malaysia, China, Bangladesh and India found that education level in diabetes patients positively associated with medication adherence. ${ }^{35,36,40,42,52}$ The studies assumed that the higher educational background of the patients might be related to their awareness and concern about medication adherence and being able to list the names of the medicines. In addition, a good level of education was associated with a patient's ability to understand the relatively complex diabetes treatment. ${ }^{54}$

Two other factors are related to the knowledge subdomain: the level of knowledge about diabetes and its medications. ${ }^{35,42,52,53}$ Adherence to medication in diabetic patients increases when these factors increase. These results confirm studies in West Asian and Middle Eastern countries with similar results. ${ }^{55,56}$ However, studies in Jordan and Pakistan showed different results that knowledge level was not associated with medication adherence in diabetic patients. ${ }^{47,57}$ This review found no negative association in education level subdomain and knowledge subdomain; however, twelve studies from seven countries concluded that education level, knowledge about diabetes, and knowledge about medications did not affect medication adherence. ${ }^{32,37-41,43-45,47,50,58}$ One of the interesting findings in the patientrelated domain was the negative association of occupational factors in one Indian study. ${ }^{33}$ In contrast, ten other studies stated that this factor was not associated with medication adherence. ${ }^{37,39-41,43-45,47,50,58}$ Detailed information about patient-related factors is summarized in Figure 3.

\section{Disease-Related Factors}

This review found five subdomains and seven specific factors to all included studies. One specific factor includes positive and negative associations with medication adherence in diabetes patients (diabetes durations). ${ }^{39,40,58}$ Research in $\mathrm{China}^{39,40}$ stated a linear association between the duration of diabetes and adherence to medication; however, research 


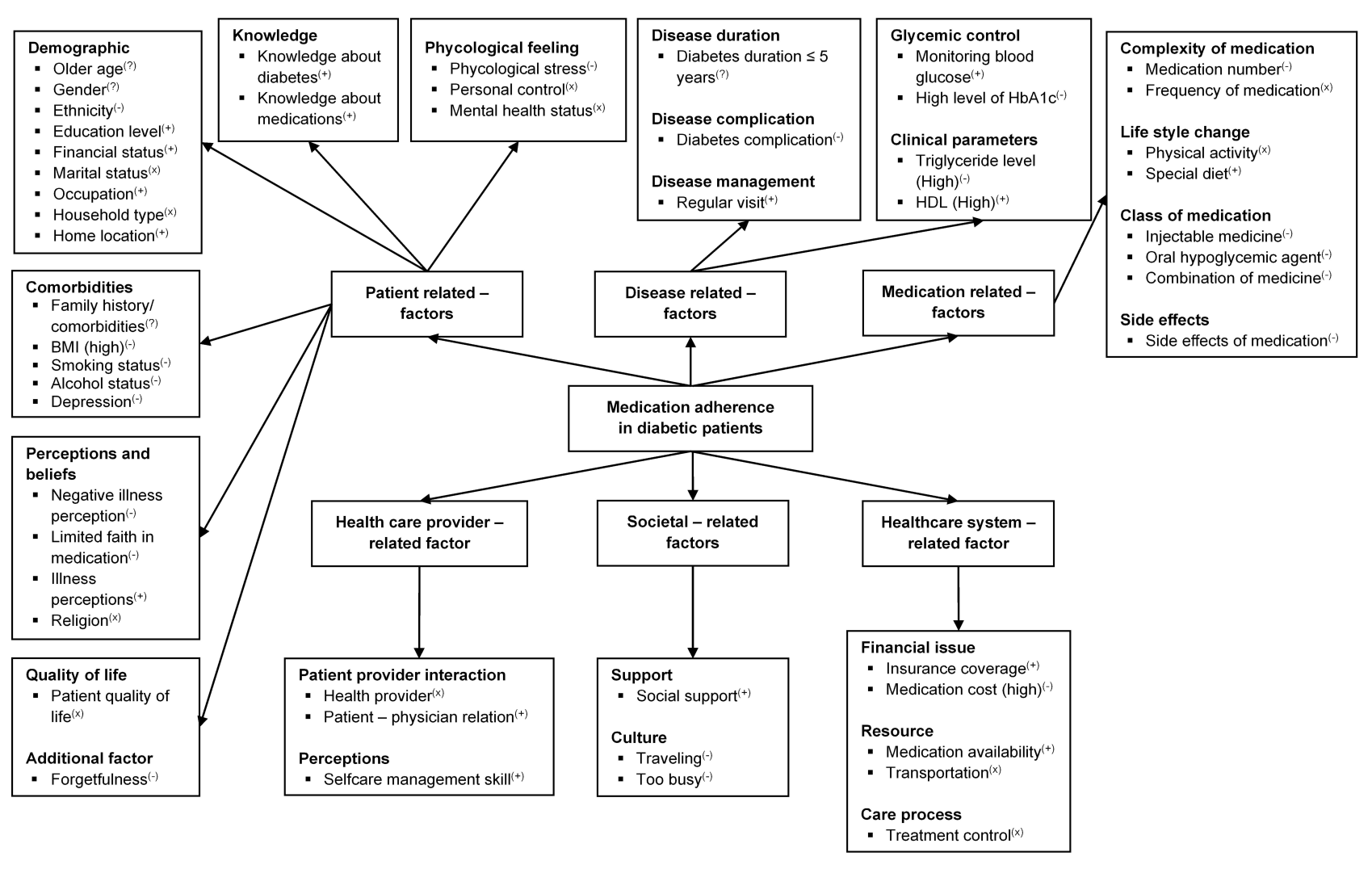

Figure 3 Conceptual framework model of medication adherence of diabetes patients in several Asian regions.

Notes: (?) Inconsistent association, the association of these factors is inconsistent with diabetes medication adherence. ${ }^{(+)}$Positive association, the association of these factors enhances or facilitates diabetes medication adherence. ${ }^{(-)}$Negative association, the association of these factors reduces or barriers to diabetes medication adherence. ${ }^{(\times)}$Nonassociated factors, these factors are not associated with medication adherence.

in Indonesia found the opposite. ${ }^{39,40,58}$ Meanwhile, ten other studies did not find an association between the duration of diabetes and adherence to medication. ${ }^{32,34,37,38,40-43,47,50}$

Other specific factors in this domain were consistently positively associated (monitoring blood glucose levels, ${ }^{31,50}$ regular visits, ${ }^{46,51}$ and high level of high-density lipoprotein ${ }^{33}$ ) or negative association (diabetes complications, ${ }^{45,46,50}$ high level of HbA1c, ${ }^{16,41,50}$ and high level of Triglyceride ${ }^{33}$ ) with medication adherence. However, a study in China and India found different facts regarding diabetes complications. ${ }^{33,34,39}$ The study concluded that diabetes complications were neither positively nor negatively associated with adherence to diabetes medication. The three studies confirmed a study in Jordan, which also concluded that diabetes complications were not associated with medication adherence. ${ }^{59}$ Other studies in Cambodia, Singapore, and Korea concluded that this factor is negatively associated with compliance. ${ }^{45,46,50}$ Detailed information about disease related factors is summarized in Figure 3.

\section{Medication-Related Factors}

There are four subdomains (complexity of medications, lifestyle change, a class of medication, and side effects) and eight specific factors summarized in medication-related domains. This review found a consistent association across all medication-specific factors associated with medication adherence. Research in Japan and Malaysia concluded that taking some diabetes medications would lower medication adherence. ${ }^{42,51}$ However, studies in West Asia (Saudi Arabia) concluded the opposite result. ${ }^{60}$ Even a recent study in Malaysia concluded that this factor was not associated with medication adherence. Meanwhile, four other studies in China, Singapore, Malaysia, Indonesia, and India found no association. ${ }^{16,34,38,58}$ Another specific factor in this domain that was negatively associated was the type of medication taken by the diabetic patient and the side effects. Four studies concluded that the use of insulin, oral hypoglycemic agents, or their combination were negatively associated with medication adherence..$^{32,33,35,40}$ Making lifestyle changes by 
following a particular diet is a specific factor that is positively related to this domain. ${ }^{46}$ Detailed information about medication-related factors is summarized in Figure 3.

\section{Health Care Provider-Related Factors}

The determinants of this domain consist of aspects related to patient-provider, knowledge, attitudes, and beliefs towards service providers and others. ${ }^{28}$ This review notes two specific domains (patient-provider interaction and patient perceptions) and three specific factors (provider, patient-physician relationship, and self-care management skills). Research in India and Bangladesh found positive associations on particular aspects of the patient-physician relationship and self-care management skills. ${ }^{31,52}$ Meanwhile, another study in India found no association with provider-specific factors. ${ }^{30}$ Health care provider factors play an increasingly important role in patient adherence to treatment for degenerative diseases. ${ }^{61}$ Detailed information about health care provider-related factors is summarized in Figure 3.

\section{Healthcare System-Related Factors}

The determinants related to the healthcare system involve three subdomains (financial issues, resources, and care processes) and five specific factors (insurance coverage, high medication cost, medication availability, transportation to a healthcare facility, and treatment control). Insurance coverage and medication availability factors were positively associated in four studies from three countries (Indonesia, China, and India). ${ }^{37,38,62}$ Meanwhile, the high medication cost factor was negatively associated in one study in Bangladesh and India. ${ }^{30,52}$ Healthcare systems determine medication adherence in several ways. For example, a limited Formulary will limit access to prescribed medications, increasing the patient's barriers to achieving optimal adherence. ${ }^{63}$ Various studies have evaluated the association of costs with diabetes and generally support the correlation of improved adherence and reduced costs. ${ }^{64,65}$ Detailed information about healthcare system-related factors is summarized in Figure 3.

\section{Societal-Related Factors}

This review notes two specific domains (support and culture) and three specific factors (social support, traveling, and being too busy) from six studies in various countries. In studies conducted in China, India, and Cambodia, specific social support factors were positively associated with medication adherence. ${ }^{31,37,46}$ However, studies conducted in India and Singapore found that these factors were not associated. ${ }^{30,40}$ Being too busy and traveling are negatively associated with adherence to taking diabetes medication. ${ }^{38}$ Detailed information about societal-related factors is summarized in Figure 3.

\section{Discussion}

This review is the first systematic review to investigate the determinants of diabetes medication adherence in South Asia, Southeast Asia, and East Asia to the best of our knowledge. Disparities in population, ethnicity, culture and socioeconomic status of diabetic patients in the region allow for differences in the determinants of medication adherence. ${ }^{19-21,66}$ This review shows that various factors are associated with adherence to diabetes medicines. These specific factors have positive, negative, or no correlations with the medication adherence of diabetic patients. Determinants of medication adherence can be classified based on the research objectives. The main objective of this review is to comprehensively classify the determinants of medication adherence for various related needs. Therefore, we classify these determinants into four categories (Figure 2). First, inconsistently factors associated with diabetes medication adherence. Four specific factors were inconsistent with diabetes medication adherence (older age, gender, diabetes duration, and family history/comorbidities). These particular factors are positively associated with medication adherence in a study. Surprisingly, they are negatively or unassociated in other studies. For example, studies in Korea and India found that old age negatively correlates with medication adherence. ${ }^{35,45}$ However, seven studies in Malaysia, China, Singapore, Japan, and India concluded that this factor was positively associated with medication adherence $(n=7) .{ }^{16,30,33,40,44,50,51}$ Most studies conclude that older patients tend to adhere to their treatment more than younger patients. Older patients tend to have more time to plan their treatment. ${ }^{30}$ Meanwhile, younger patients are still working as employees, so that the tight working hours may cause their non-adherence. ${ }^{16}$ 
A study in Korea found that the female gender was positively associated with medication adherence. ${ }^{45}$ However, these factors were negatively associated with adherence in a study in China and India. ${ }^{33,39}$ The disease duration factor $(\leq$ 5 years) was positively associated in a study in China and India. ${ }^{33,40}$ However, recent studies conducted in China, Indonesia, and even India concluded that this factor was negatively associated with medication adherence. ${ }^{35,39,58}$ As the disease progresses, diabetic patients may gain increased knowledge about diabetes and its treatment through information about health care providers. This increase makes patients aware of the need for adherence in diabetes treatment. Another systematic review also found the inconsistency of associations between several determinants of medication adherence. ${ }^{13,67,68}$ Differences in places, conditions, subjects, research time, and measurement techniques may cause the inconsistency of factors influencing medication adherence. Further study is required to investigate these three variables' processes on medication adherence in diabetes patients.

Second, positively associated factors with diabetes medication adherence (Figure 2). Sixteen specific factors were found to be positively associated in this review. Several studies have found that five factors are more dominant in this category. These factors are the level of education, ${ }^{35,36,40,42,52}$ knowledge about diabetes and its medications, ${ }^{35,42,52,53}$ medication availability, ${ }^{36,38,62}$ social support, ${ }^{31,37,46}$ and socioeconomic status. ${ }^{31,32,35,44,46}$ More than half of the studies in this review found these four factors to be positively associated $(n=16)$. Six studies concluded that education and knowledge about diabetes or its treatment were positively associated with medication adherence $(n=6) .{ }^{32,35,40,42,52,53}$ This association can help patients understand the doctor's explanation so that the patient's awareness and attention appear to comply with his treatment. ${ }^{42,69}$ Social support factors were explicitly studied in China, ${ }^{37}$ however, it is corroborated by two other studies in India and Cambodia. ${ }^{31,46}$ Diabetic patients who receive social support are more likely to feel psychologically comfortable and positively deal with their health problems. ${ }^{37,70}$ An excellent financial level in diabetic patients also positively affects their treatment. Five studies in Cambodia, ${ }^{46}$ India, ${ }^{31,32,35}$ and Malaysia ${ }^{44}$ concluded that better financial status allows patients to have more freedom in seeking health $(n=5) .{ }^{31,32,35,44,46}$

Third, negatively associated factors with diabetes medication adherence (Figure 2). This review found twenty-one factors identified as negative factors. More than ten studies included in this review found that negatively associated factors were in the domain of patient-related factors. Five specific factors dominate in this category. The five factors are negative illness perceptions, ${ }^{38,39,43}$ little faith in medications, ${ }^{38,53,62}$ forgetfulness, ${ }^{38,52,62}$ diabetes complications, ${ }^{45,46,50}$ and a high level of HbAlc. ${ }^{16,41,50}$ Possible explanations for each of these factors are: (1) the symptoms of diabetes tend to be felt more severe by patients who have negative perceptions and do not accept their disease; ${ }^{38,43}$ (2) limited belief in drugs prescribed by doctors and concerns with side effects; ${ }^{38,53,62}$ (3) diabetic patients who are under stress tend to forget their various treatment instructions, ${ }^{38,53,62}$ (4) the emergence of diabetes complications makes the treatment more complex so that patients are confused and choose not to take it. ${ }^{45,46,50}$ These reasons may underlie the patient skipping or increasing the dose of the medicines.

Fourth, factors are not associated with diabetes medication adherence (Figure 2). This category refers to neither positively nor negatively associated factors in all included studies. Marital status is the only dominant factor in this category studied by most studies. ${ }^{16,30,32,34,35,37,39,41,43,45,47,50}$ This finding confirms a previous review conducted in the African region that marital status is not associated with medication adherence. ${ }^{13}$ Other factors studied by more than one study are frequency of medications, physical activity, and transportation to a health care facility. ${ }^{16,30,34,38,44,45}$ However, other studies have stated that this factor is negatively associated with adherence. ${ }^{59,71}$ This difference is common in studies of medication adherence determinants in various diseases. ${ }^{72}$ In addition to differences in the study population, the study site, the severity of each patient, socio-cultural and local economic conditions may also affect the variability of the results. ${ }^{19-21}$

Almost all studies found factors that were not associated with diabetes medication adherence. For example, the medication number factor (polypharmacy) was negatively associated in studies in Japan and Malaysia. ${ }^{42,51}$ However, five other studies in China, ${ }^{38}$ Singapore, ${ }^{16}$ Malaysia, ${ }^{41}$ Indonesia, ${ }^{41}$ and India $^{34}$ concluded that the medication number (polypharmacy) was not an associated factor with medication adherence. Another example is the social support factor studied by five studies in China, India, Cambodia, and Singapore. ${ }^{30,31,37,46,50}$ Three of these studies state that social support factors are positively associated. ${ }^{31,37,46}$ However, two other studies found no such association. ${ }^{30,50}$ The determinants of diabetes treatment adherence are considered complex because the results of studies can vary. ${ }^{27,68}$ 
This review confirms previous research that the facilitating and barriers for diabetes medication adherence are influenced by various complex factors. ${ }^{13,68,72}$ A study can conclude that certain specific factors may be positively, negatively, or unassociated with medication adherence (Figure 2). However, other studies may find otherwise. Most studies found the domain of patient-related determinants to dominate the association with medication adherence. This domain relates to the patient's specific demographics, physiological feelings, knowledge, perceptions and beliefs, comorbidities, quality of life, and other factors related to the patient. Several studies have concluded that this domain can predict diabetes medication adherence. ${ }^{73}$ This indicates that health care providers must be aware that these dominant factors can be the basis for developing intervention strategies to maximize therapeutic success.

The definition of medication adherence in this review cannot be generalized because of different types of adherence scales. Globally, several medication adherence scales are available to assess medication adherence in individuals with diabetes. ${ }^{48}$ However, only a few scales have been designed to assess medication adherence for individuals with diabetes. Most studies used the MMAS- 8 scale, which was validated by other studies in different settings and populations from the population studied. ${ }^{16,30-32,34,36,39-41,43,44,46,47,53,62,74}$ The MMAS- 8 was created and developed as a medication adherence scale for hypertensive patients, so validation studies through psychometric testing are needed before being used in other disease populations. ${ }^{48,75}$ Psychometric tests on a measurement scale are still needed because the characteristics of the questions, responses to the scale, population, context, conditions, and timing of measurements can affect the results. ${ }^{76-78}$ Inappropriate medication adherence scales will affect the final interpretation and may bias patients' tendencies to overreport to protect themselves from guilt due to failure to comply with medication recommendations. The psychometric test will increase the accuracy of its measurement because each scale is created and validated in a specific population. ${ }^{48,78}$ Another possible alternative is to design and develop a particular medication adherence scale for specific people and diseases based on the determinants of adherence.

In this review, the medication adherence rate among diabetic patients was low to moderate. Most studies found that the biological marker of blood glucose (HbAlc) exceeded the standard value $(n=62.9 \%)$. Several developing countries and other Asian regions also found medication adherence in the low-medium category with relatively high glucose levels in people with diabetes. ${ }^{12-14}$ One of the reasons why blood glucose targets are challenging to achieve could be the misconception that diabetes is not a severe disease. ${ }^{79}$ This condition is a warning for health stakeholders in the Asian region to be aware of the adverse effects of non-adherence to treatment in diabetic patients. Meanwhile, The government will be impacted through increasing national health financing because the cost of treating diabetes patients with complications will increase. ${ }^{17}$

Understanding the determinants of medication adherence in the four classifications and the conceptual framework of this review will benefit patients, health care providers, and governments. This review can support the development of more specific adherence scales in diabetic patients in the Asian region based on their adherence predictors. ${ }^{73,80,81}$ Stakeholders can develop intervention strategies to improve diabetes medication adherence based on the findings of this review. The intervention can prioritize the dominant factor in classifying factors with positive or negative associations. Educational factor intervention increases patient knowledge about diabetes and its treatment. ${ }^{54,69,82}$ These interventions can be carried out by any health professional who serves diabetes patients, such as doctors, pharmacists, nurses, public health officers, and others. This intervention can be performed in most health care settings because of the relatively low resource requirements and ease of implementation. ${ }^{54,69}$ A randomized controlled trial study in Iran used two educational strategies, re-education and illustrated pictures, to increase knowledge about diabetes in diabetic patients with low literacy. ${ }^{82}$ This review examines medication adherence factors from studies conducted in different countries. However, it must be acknowledged that differences in health systems, cultures, and beliefs pose a challenge to generalize the relationship between each factor. Several limitations in this systematic review should be considered carefully.

Limitations on including scientific articles in English may be an obstacle to obtaining a more comprehensive range of research articles. We may miss out on some potentially relevant studies in other languages. The use of CCAT for critical appraisal of an article depends on the ratter's judgment. It is possible to ignore performance in individual categories and focus only on the total score. Therefore, the assessment was carried out at least by two research members to reduce this limitation. Most studies used other disease self-report adherence scales without conducting validity tests or psychometric tests in the diabetic population. These constraints may increase the risk of bias and restrict the generalizability of the 
results. A mixed-method study design is needed to determine why and how these determinants affect medication adherence and a meta-analysis study to obtain more substantial evidence. However, a systematic approach using rigorous standardized methods has ensured the quality of these systematic reviews. ${ }^{22,23,26}$

\section{Conclusion}

This research can be an essential foundation for various goals related to medication adherence of diabetic patients. Most of the determinants showed varying statistical significance and direction of their effects on diabetes medication adherence. Patient-related factors dominate as positively or negatively associated factors in several Asian regions. Policymakers and health care providers need to tailor interventions and specifically target improving education levels, knowledge about diabetes and its treatment, drug availability, social support, and socioeconomic appropriate for diabetes patients in this Asian region. These determinants of medication adherence need to be evaluated further to provide more substantial evidence of each factor through a meta-analysis or mixed-method study. Future studies need to explore alternative scales or methods to assess medication adherence or develop a scale for evaluating diabetes medication adherence with higher reliability and validity, especially in low literacy populations.

\section{Acknowledgments}

The authors would like to thank the Indonesia Endowment Fund for Education (BUDI DN and LPDP) for supporting this systematic review.

\section{Disclosure}

The authors report no conflicts of interest in this work. The funder has no involvement or control in the research design, analysis, or interpretation of data, including in scriptwriting or the choice to publish the findings.

\section{References}

1. Williams R, Colagiuri S, Almutairi R, et al. IDF Diabetes Atlas. 9th ed. International Diabetes Federation; 2019.

2. Siegel KR, Patel SA, Ali MK. Non-communicable diseases in South Asia: contemporary perspectives. Br Med Bull. 2014;111(1):31-44. doi:10.1093/bmb/ldu018

3. Putri SMNK, Christina M, Saverinus S, Halimatunnisa H, Susanti S. Factors associated with obesity in adults in South East Asia. Int J Psychosoc Rehabilitation. 2020;24(7):54.

4. Low WY, Lee YK, Samy AL. Non-communicable diseases in the Asia-Pacific region: prevalence, risk factors and community-based prevention. IJOMEH. 2014;28(1). doi:10.2478/s13382-014-0326-0

5. Mannava P, Abdullah A, James C, Dodd R, Annear PL. Health systems and noncommunicable diseases in the Asia-Pacific Region: a review of the published literature. Asia Pac J Public Health. 2015;27(2):NP1-NP19. doi:10.1177/1010539513500336

6. Chaudhury A, Duvoor C, Reddy Dendi VS, et al. Clinical review of antidiabetic drugs: implications for type 2 diabetes mellitus management. Front Endocrinol. 2017;8(JAN). doi:10.3389/fendo.2017.00006

7. Verhulst MJL, Loos BG, Gerdes VEA, Teeuw WJ. Evaluating all potential oral complications of diabetes mellitus. Front Endocrinol (Lausanne). 2019;10(56). doi:10.3389/fendo.2019.00056

8. Meddings J, Kerr EA, Heisler M, Hofer TP. Physician assessments of medication adherence and decisions to intensify medications for patients with uncontrolled blood pressure: still no better than a coin toss. BMC Health Serv Res. 2012;12(1):1. doi:10.1186/1472-6963-12-270

9. Winters A, Esse T, Bhansali A, Serna O, Mhatre S, Sansgiry S. Physician perception of patient medication adherence in a cohort of medicare advantage plans in Texas. J Manag Care Spec Pharm. 2016;22(3):305-312. doi:10.18553/jmcp.2016.22.3.305

10. Currie CJ, Peyrot M, Morgan CL, et al. The impact of treatment noncompliance on mortality in people with type 2 diabetes. Diabetes Care. 2012;35(6):1279-1284. doi:10.2337/dc11-1277

11. Brown MT, Bussell J, Dutta S, Davis K, Strong S, Mathew S. Medication adherence: truth and consequences. Am J Med Sci. 2016;351(4):387-399. doi:10.1016/j.amjms.2016.01.010

12. Krass I, Schieback P, Dhippayom T. Adherence to diabetes medication: a systematic review. Diabet Med. 2015;32(6):725-737. doi:10.1111/ dme. 12651

13. Jaam M, Ibrahim MIM, Kheir N, Awaisu A. Factors associated with medication adherence among patients with diabetes in the Middle East and North Africa region: a systematic mixed studies review. Diabetes Res Clin Pract. 2017;129:1-15. doi:10.1016/j.diabres.2017.04.015

14. Al-lela OQB, Abdulkareem RA, AL-Mufti L, Kamal N, Qasim S, Sagvan R. Medication adherence among diabetic patients in developing countries: review of studies. Syst Rev Pharm. 2020;11(8):6.

15. Dana A, Lara M, Maya EH, et al. Evaluation of antidiabetic medication adherence in the Lebanese population: development of the Lebanese diabetes medication adherence scale. Int J Pharm Pract. 2019;156:107837.

16. Lee CS, Tan JHM, Sankari U, Koh YLE, Tan NC. Assessing oral medication adherence among patients with type 2 diabetes mellitus treated with polytherapy in a developed Asian community: a cross-sectional study. BMJ Open. 2017;7(9):e016317. doi:10.1136/bmjopen-2017-016317 
17. Seuring T, Archangelidi O, Suhrcke M. The economic costs of type 2 diabetes: a global systematic review. PharmacoEconomics. 2015;33 (8):811-831. doi:10.1007/s40273-015-0268-9

18. Al Hamid A, Ghaleb M, Aljadhey H, Aslanpour Z. A systematic review of qualitative research on the contributory factors leading to medicine-related problems from the perspectives of adult patients with cardiovascular diseases and diabetes mellitus. BMJ Open. 2014;4(9): e005992-e005992. doi:10.1136/bmjopen-2014-005992

19. Alzubaidi H, Mc Narmara K, Kilmartin GM, Kilmartin JF, Marriott J. The relationships between illness and treatment perceptions with adherence to diabetes self-care: a comparison between Arabic-speaking migrants and Caucasian English-speaking patients. Diabetes Res Clin Pract. $2015 ; 110$ (2):208-217. doi:10.1016/j.diabres.2015.08.006

20. Shahin W, Kennedy GA, Stupans I. The impact of personal and cultural beliefs on medication adherence of patients with chronic illnesses: a systematic review. Patient Prefer Adherence. 2019;13:1019-1035. doi:10.2147/PPA.S212046

21. Widayanti AW, Norris P, Heydon S, Green JA. Medicine taking behaviours of people with type 2 diabetes in Indonesia: a qualitative study. Int J Clin Pharm. 2020;42(1):31-39. doi:10.1007/s11096-019-00933-0

22. Page MJ, McKenzie JE, Bossuyt PM, et al. The PRISMA 2020 statement: an updated guideline for reporting systematic reviews. PLoS Med. 2021;18(3):e1003583. doi:10.1371/journal.pmed.1003583

23. Bramer WM, Rethlefsen ML, Kleijnen J, Franco OH. Optimal database combinations for literature searches in systematic reviews: a prospective exploratory study. Syst Rev. 2017;6(1):245. doi:10.1186/s13643-017-0644-y

24. Eriksen MB, Frandsen TF. The impact of patient, intervention, comparison, outcome (PICO) as a search strategy tool on literature search quality: a systematic review. jmla. 2018;106(4). doi:10.5195/JMLA.2018.345

25. Hopewell S, Clarke MJ, Lefebvre C, Scherer RW. Handsearching versus electronic searching to identify reports of randomized trials. Cochrane Methodology Review Group, ed. Cochrane Database Syst Rev. 2007;2010(1). doi:10.1002/14651858.MR000001.pub2

26. Crowe M, Sheppard L, Campbell A. Comparison of the effects of using the Crowe Critical Appraisal Tool versus informal appraisal in assessing health research: a randomised trial. Int J Evid Based Healthc. 2011;9(4):444-449. doi:10.1111/j.1744-1609.2011.00237.x

27. Jaam M, Awaisu A, Mohamed Ibrahim MI, Kheir N. A holistic conceptual framework model to describe medication adherence in and guide interventions in diabetes mellitus. Res Social Adm Pharm. 2018;14(4):391-397. doi:10.1016/j.sapharm.2017.05.003

28. Sabate E. Adherence to long-term therapies: evidence for action. World Health Organization; 2003.

29. Lee YH. An overview of meta-analysis for clinicians. Korean J Intern Med. 2018;33(2):277-283. doi:10.3904/kjim.2016.195

30. Mentock SM, Ng VY, Narayana R, et al. Treatment-seeking behavior and obstacles to treatment compliance in diabetic patients in Mangaluru, India. Diabetes Metab Syndr. 2017;11(Suppl 2):S617-S622. doi:10.1016/j.dsx.2017.04.014

31. Sankar UV, Lipska K, Mini GK, Sarma PS, Thankappan KR. The adherence to medications in diabetic patients in rural Kerala, India. Asia Pac J Public Health. 2015;27(2):513-523. doi:10.1177/1010539513475651

32. Basu S, Khobragade M, Kumar A, Raut DK. Medical adherence and its predictors in Diabetes Mellitus patients attending government hospitals in the Indian Capital, Delhi, 2013: a cross sectional study. Int J Diabetes Dev Ctries. 2015;35(S2):95-101. doi:10.1007/s13410-014-0232-9

33. Haghighatpanah M, Nejad ASM, Haghighatpanah M, Thunga G, Mallayasamy S. Factors that correlate with poor glycemic control in Type 2 diabetes mellitus patients with complications. Osong Public Health Res Perspect. 2018;9(4):167-174. doi:10.24171/j.phrp.2018.9.4.05

34. Thapar R, Holla R, Kumar N, et al. Factors influencing adherence to anti-diabetes medications among type 2 diabetes patients attending tertiary care hospitals in Mangaluru. Clin Epidemiology Glob Health. 2020;8(4):1089-1093. doi:10.1016/j.cegh.2020.03.025

35. Mukherjee S, Sharmasarkar B, Das KK, Bhattacharyya A, Deb A. Compliance to anti-diabetic drugs: observations from the diabetic clinic of a Medical College in Kolkata, India. JCDR. 2013;7(4):661-665. doi:10.7860/JCDR/2013/5352.2876

36. Basu S, Garg S, Sharma N, Singh MM, Garg S. Adherence to self-care practices, glycemic status and influencing factors in diabetes patients in a tertiary care hospital in Delhi. WJD. 2018;9(5):72-79. doi:10.4239/wjd.v9.i5.72

37. Gu L, Wu S, Zhao S, et al. Association of social support and medication adherence in Chinese patients with Type 2 diabetes mellitus. Int J Environ Res Public Health. 2017;14(12):1522. doi:10.3390/ijerph14121522

38. Wu P, Liu N. Association between patients' beliefs and oral antidiabetic medication adherence in a Chinese type 2 diabetic population. Patient Prefer Adherence. 2016;10:1161-1167. doi:10.2147/PPA.S105600

39. Xu N, Xie S, Chen Y, Li J, Long S. Factors influencing medication non-adherence among Chinese older adults with diabetes mellitus. Int J Environ Res Public Health. 2020;17(17):6012. doi:10.3390/ijerph17176012

40. Zhang Y, Ting RZ, Yang W, et al. Depression in Chinese patients with type 2 diabetes: associations with hyperglycemia, hypoglycemia, and poor treatment adherence. J Diabetes. 2015;7(6):800-808. doi:10.1111/1753-0407.12238

41. Abdullah NF, Khuan L, Theng CA, Sowtali SN, Juni MH. Effect of patient characteristics on medication adherence among patients with type 2 diabetes mellitus: a cross-sectional survey. Contemp Nurse. 2019;55(1):27-37. doi:10.1080/10376178.2019.1583067

42. Al-Qazaz H, Sulaiman SA, Hassali MA, et al. Diabetes knowledge, medication adherence and glycemic control among patients with type 2 diabetes. Int J Clin Pharm. 2011;33(6):1028-1035. doi:10.1007/s11096-011-9582-2

43. Balasubramaniam S, Lim SL, Goh LH, Subramaniam S, Tangiisuran B. Evaluation of illness perceptions and their associations with glycaemic control, medication adherence and chronic kidney disease in type 2 diabetes mellitus patients in Malaysia. Diabetes Metab Syndr. 2019;13 (4):2585-2591. doi:10.1016/j.dsx.2019.07.011

44. Chew BH, Hassan NH, Sherina MS. Determinants of medication adherence among adults with type 2 diabetes mellitus in three Malaysian public health clinics: a cross-sectional study. PPA. 2015;639. doi:10.2147/PPA.S81612

45. Kim H, Kim HS, Bowman JD, Cho NH. Comparing diabetic patient characteristics related to stated medication adherence in a rural vs. urban community in Korea. J Clin Pharm Ther. 2016;41(1):40-46. doi:10.1111/jcpt.12344

46. Nonogaki A, Heang H, Yi S, et al. Factors associated with medication adherence among people with diabetes mellitus in poor urban areas of Cambodia: a cross-sectional study. PLoS One. 2019;14(11):1-17. doi:10.1371/journal.pone.0225000

47. Nazir SUR, Hassali MA, Saleem F, Bashir S, Aljadhey H. Disease related knowledge, medication adherence and glycaemic control among patients with type 2 diabetes mellitus in Pakistan. Prim Care Diabetes. 2015;10(2):136-141. doi:10.1016/j.pcd.2015.09.004

48. Wibowo MINA, Yasin NM, Kristina SA, Prabandari YS. A systematic review on self-reported questionnaires to assess medication adherence in diabetic patients. Malaysian J Public Health Med. 2021;21(2):359-373. doi:10.37268/mjphm/vol.21/no.2/art.1048 
49. Nguyen TMU, Caze AL, Cottrell N. What are validated self-report adherence scales really measuring?: a systematic review: systematic review on validated medication adherence measurement scales. Br J Clin Pharmacol. 2014;77(3):427-445. doi:10.1111/bcp.12194

50. Zhang ZP, Premikha M, Luo M, Venkataraman K. Diabetes distress and peripheral neuropathy are associated with medication non-adherence in individuals with type 2 diabetes in primary care. Acta Diabetol. 2020;58(3):309-317. doi:10.1007/s00592-020-01609-2

51. Horii T, Momo K, Yasu T, Kabeya Y, Atsuda K. Determination of factors affecting medication adherence in type 2 diabetes mellitus patients using a nationwide claim-based database in Japan. PLoS One. 2019;14(10):1-12. doi:10.1371/journal.pone.0223431

52. Islam SMS, Biswas T, Bhuiyan FA, Mustafa K, Islam A. Patients' perspective of disease and medication adherence for type 2 diabetes in an urban area in Bangladesh: a qualitative study. BMC Res Notes. 2017;10(131). doi:10.1186/s13104-017-2454-7

53. Hussain N, Said ASA, Khan Z. Influence of health literacy on medication adherence among elderly females with Type 2 diabetes in Pakistan. Int Q Community Health Educ. 2020;41(1):35-44. doi:10.1177/0272684X19896724

54. Lorenzi GM, LaRue SM, Collins SE. Effects of a patient education support program on pramlintide adherence. Clin Diabetes. $2011 ; 29(1): 17-24$. doi:10.2337/diaclin.29.1.17

55. Jeragh-Alhaddad F, Waheedi M, Barber N, Brock T. Barriers to medication taking among Kuwaiti patients with type 2 diabetes: a qualitative study. Patient Prefer Adherence. 2015;1491. doi:10.2147/PPA.S86719

56. Sweileh WM, Zyoud SH, Abu Nab'a RJ, et al. Influence of patients' disease knowledge and beliefs about medicines on medication adherence: findings from a cross-sectional survey among patients with type 2 diabetes mellitus in Palestine. BMC Public Health. 2014;14(1):94. doi:10.1186/ 1471-2458-14-94

57. Mada'en S, Elayeh E, Akour A, AlQhaiwi T, Shaggour B, Madain R. Diabetes knowledge, medication adherence, and glycemic control among diabetic patients: a cross-sectional study in Jordan. J App Pharm Sci. 2020;10(4):41-46. doi:10.7324/JAPS.2020.104007

58. Wulandari N, Maifitrianti M, Hasanah F, Atika S, Dini putri R. Medication adherence assessment among patients with type 2 diabetes mellitus treated polytherapy in Indonesian community health center: a cross sectional-study. J Pharm Bioall Sci. 2020;12(6):758. doi:10.4103/jpbs. JPBS_257_19

59. Jarab AS, Almrayat R, Alqudah S, et al. Predictors of non-adherence to pharmacotherapy in patients with type 2 diabetes. Int J Clin Pharm. 2014;36:9. doi:10.1007/s11096-014-9938-5

60. Khan A, Al-Abdul Lateef Z, Al Aithan M, Bu-Khamseen M, Al Ibrahim I, Khan S. Factors contributing to non-compliance among diabetics attending primary health centers in the Al Hasa district of Saudi Arabia. J Fam Community Med. 2012;19(1):26. doi:10.4103/2230-8229.94008

61. Bussell JK, Cha E, Grant YE, Schwartz DD, Young LA. Ways health care providers can promote better medication adherence. Clin Diabetes. 2017;35(3):171-177. doi:10.2337/cd016-0029

62. Aditama L, Athiyah U, Utami W, Rahem A. Adherence behavior assessment of oral antidiabetic medication use: a study of patient decisions in long-term disease management in primary health care centers in Surabaya. J Basic Clin Physiol Pharmacol. 2020;30(6). doi:10.1515/jbcpp-20190257

63. Braithwaite S, Shirkhorshidian I, Jones K, Johnsrud M. The role of medication adherence in the U.S. healthcare system. Sargas Pharm Adherence Compliance. 2013. Available from: https://spacinternational.com/pdf/adher.pdf.

64. Iuga AO, McGuire MJ. Adherence and health care costs. RMHP. 2014;35. doi:10.2147/RMHP.S19801

65. Stuart B, Davidoff A, Lopert R, Shaffer T, Samantha Shoemaker J, Lloyd J. Does medication adherence lower medicare spending among beneficiaries with diabetes?: Does medication adherence lower medicare spending? Health Serv Res. 2011;46(4):1180-1199. doi:10.1111/j.14756773.2011.01250.x

66. Egede LE, Lynch CP, Gebregziabher M, et al. Differential impact of longitudinal medication non-adherence on mortality by race/ethnicity among veterans with diabetes. J GEN INTERN MED. 2013;28(2):208-215. doi:10.1007/s11606-012-2200-8

67. Aminde LN, Tindong M, Ngwasiri CA, et al. Adherence to antidiabetic medication and factors associated with non-adherence among patients with type-2 diabetes mellitus in two regional hospitals in Cameroon. BMC Endocr Disord. 2019;19(1):35. doi:10.1186/s12902-019-0360-9

68. Gast A, Mathes T. Medication adherence influencing factors—an (updated) overview of systematic reviews. Syst Rev. 2019;8(1):112. doi:10.1186/ s13643-019-1014-8

69. Taibanguay N, Chaiamnuay S, Asavatanabodee P, Narongroeknawin P. Effect of patient education on medication adherence of patients with rheumatoid arthritis: a randomized controlled trial. PPA. 2019;13:119-129. doi:10.2147/PPA.S192008

70. Scheurer D, Choudhry N, Swanton KA, Matlin O, Shrank W. Association between different types of social support and medication adherence. Am J Manag Care. 2012;18(12):e461-467.

71. Farsaei S, Radfar M, Heydari Z, Abbasi F, Qorbani M. Insulin adherence in patients with diabetes: risk factors for injection omission. Prim Care Diabetes. 2014;8(4):338-345. doi:10.1016/j.pcd.2014.03.001

72. Dhar L, Dantas J, Ali M. A systematic review of factors influencing medication adherence to hypertension treatment in developing countries. OJEpi. 2017;07(03):211-250. doi:10.4236/ojepi.2017.73018

73. Demoz GT, Wahdey S, Bahrey D, et al. Predictors of poor adherence to antidiabetic therapy in patients with type 2 diabetes: a cross-sectional study insight from Ethiopia. Diabetol Metab Syndr. 2020;12(1):62. doi:10.1186/s13098-020-00567-7

74. Rathish D, Hemachandra R, Premadasa T, et al. Comparison of medication adherence between type 2 diabetes mellitus patients who pay for their medications and those who receive it free: a rural Asian experience. J Health Popul Nutr. 2019;38(1):4. doi:10.1186/s41043-019-0161-9

75. Morisky DE, Ang A, Krousel-Wood M, Ward HJ. Predictive validity of a medication adherence measure in an outpatient setting. $J$ Clin Hypertens. 2008;10(5):348-354. doi:10.1111/j.1751-7176.2008.07572.x

76. Chan EKH, Zumbo BD, eds. Validity and Validation in Social, Behavioral, and Health Sciences. 1st ed. Springer International Publishing: Imprint: Springer; 2014. doi:10.1007/978-3-319-07794-9

77. Costa LD, Remedios R. Different methods, different results: examining the implications of methodological divergence and implicit processes for achievement goal research. J Mix Methods Res. 2014;8(2):162-179. doi:10.1177/1558689813495977

78. Wild D, Grove A, Martin M, et al. Principles of good practice for the translation and cultural adaptation process for patient-reported outcomes (PRO) measures: report of the ISPOR task force for translation and cultural adaptation. Value Health. 2005;8(2):94-104. doi:10.1111/j.15244733.2005.04054.x

79. Kalra S, Jena B, Yeravdekar R. Emotional and psychological needs of people with diabetes. Indian J Endocr Metab. 2018;22(5):696. doi:10.4103/ ijem.IJEM_579_17 
80. Kripalani S, Risser J, Gatti ME, Jacobson TA. Development and evaluation of the adherence to refills and medications scale (ARMS) among low-literacy patients with chronic disease. Value Health. 2009;12(1):118-123. doi:10.1111/j.1524-4733.2008.00400.x

81. Naqvi AA, Hassali MA, Rizvi M, et al. Development and validation of a novel general Medication Adherence Scale (GMAS) for chronic illness patients in Pakistan. Front Pharmacol. 2018;9:1124. doi:10.3389/fphar.2018.01124

82. Negarandeh R, Mahmoodi H, Noktehdan H, Heshmat R, Shakibazadeh E. Teach back and pictorial image educational strategies on knowledge about diabetes and medication/dietary adherence among low health literate patients with type 2 diabetes. Prim Care Diabetes. 2013;7(2):111-118. doi:10.1016/j.pcd.2012.11.001

\section{Publish your work in this journal}

Patient Preference and Adherence is an international, peer-reviewed, open access journal that focusing on the growing importance of patient preference and adherence throughout the therapeutic continuum. Patient satisfaction, acceptability, quality of life, compliance, persistence and their role in developing new therapeutic modalities and compounds to optimize clinical outcomes for existing disease states are major areas of interest for the journal. This journal has been accepted for indexing on PubMed Central. The manuscript management system is completely online and includes a very quick and fair peer-review system, which is all easy to use. Visit http://www.dovepress.com/testimonials.php to read real quotes from published authors.

Submit your manuscript here: https://www.dovepress.com/patient-preference-and-adherence-journal 